\title{
Eigenleistungen, Zeit und Unabhängigkeit ${ }^{1}$
}

\author{
Jiř́i Skolka
}

Während der Industrialisierung der letzten 200 Jahre ging die Haushaltsproduktion für den Eigenbedarf zurück. Gegenwärtig zeichnet sich allerdings in den Industriestaaten eine gewisse Renaissance der Eigenleistungen der Haushalte ab. Diese Wende hat vielfältige wirtschaftliche, technologische und gesellschaftliche Gründe.

\section{Industrialisierung und Eigenleistungen}

Die Industrialisierung - die „Große Transformation“ (Polanyi, 1944) der traditionellen Gesellschaft in eine industrielle - hat weitgehende Arbeitsteilungen ${ }^{2}$ in der Organisation der Produktion und im sozialen Leben hervorgerufen (Skolka, 1985):

- Der Zeitaufwand für produktive ${ }^{3}$ Arbeit verschob sich zugunsten der bezahlten erwerbstätigen Arbeit ${ }^{4}$; unentgeltliche Eigenleistungen gingen zurück. Es wurde mehr für den Markt und weniger für den Eigenbedarf produziert. Erwerbstätige Arbeit, Arbeit für den Eigenbedarf und Freizeit wurden voneinander scharf getrennt.

- Aus Produzenten für den Eigengebrauch sind Erzeuger von Gütern für andere und Konsumenten von Gütern, die andere erzeugt haben, geworden; die Arbeit wurde ,entfremdet“.

- In der traditionellen Wirtschaft wurde überwiegend für den laufenden Verbrauch produziert. In der Industriewirtschaft werden umfangreiche Investitionen in Produktionseinrichtungen und Verkehrsmittel getätigt; die marktmäßige Produktion teilt sich in Produktion kurzlebiger Verbrauchsgüter und langlebiger Investitionsgüter.

- Geldloser Tausch der Verbrauchsgüter wurde durch Verkauf von Waren und Dienste für Geld ersetzt; Geld dient auch der Akkumula- 
tion des Finanzkapitals für die Beschaffung der Investitionen. Die monetäre Bewertung der Produktion für den Markt hat die traditionelle Bewertung der Waren und Dienste nach den Kriterien der „moral economy“ (Thompson, 1971) zurückgedrängt.

- Produktive Tätigkeiten erwiesen sich als unterschiedlich innovationsfähig. Heute können sie teils einem innovationsintensiven (fortschrittlichen), teils einem innovationsarmen (rückständigen) Sektor zugeordnet werden. Unterschiede in der Innovationsintensität beeinflußten Beschäftigung, Produktivität und Preise in beiden Sektoren (Baumol, 1967).

Neben diesen „Makro-Spaltungen“ der Wirtschaft und Gesellschaft kam es zu Diversifizierungen auf der „Mikro-Ebene“. Die Anzahl der Waren und Dienstleistungen, der wirtschaftlichen Aktivitäten und Berufe nahm zu.

Obwohl während der zwei Jahrhunderte der Industrialisierung die erwerbstätige Arbeit kräftig stieg, beansprucht heute in den entwickelten Ländern die Eigenarbeit noch etwa die Hälfte der produktiven Arbeitszeit (Goldsmith-Clermont, 1982). In den Haushalten gibt es weiterhin Tätigkeiten, deren Qualifikationsansprüche niedrig sind, in denen Skalenerträge nicht erreicht werden können, deren qualitative und emotionelle Aspekte aber bedeutend sind. Nach Vollendung der Industrialisierung, beim Übergang zur Dienstleistungsgesellschaft, kommt die Tendenz zugunsten der erwerbstätigen Arbeit zum Stillstand, und in den Industrieländern beginnt eine Renaissance der Eigenleistungen der Haushalte.

\section{Renaissance der Eigenleistungen in Österreich}

Die Wiederbelebung der Eigenproduktion der Haushalte in Österreich während der letzten Jahrzehnte kann mit statistischen Daten zwar nicht systematisch, für viele Tätigkeiten dennoch ausreichend belegt werden (Skolka, 1986):

- Personenverkehr: Der Individualverkehr hat in der Nachkriegszeit den öffentlichen Verkehr verdrängt. Die Ausstattung der Haushalte mit Personenkraftwagen stieg von 13 Autos je 1000 Personen 1954 auf 215 Autos 1974 und 323 Autos 1984 (Wüger, 1981); 1974 besaßen 49 Prozent, 197955 Prozent und 198461 Prozent der Haushalte Personenkraftwagen (Vocelka-Zeidler, 1985). 1980 wurden im Individualverkehr rund 55 Milliarden Personenkilometer erbracht, d. h. fast 78 Prozent aller Personenverkehrsleistungen auf Schiene und Straße. Die geschätzte (zugerechnete) Wertschöpfung des Individualverkehrs war 1980 mit etwa 62 Milliarden Schilling gleich hoch wie die Wertschöpfung des gesamten institutionellen Verkehrs (Puwein, 1984). Der Anteil der privaten Autos an den gesamten Leistungen des Personenverkehrs (in Personenkilometern) hat sich von 54 Prozent 1960 auf 74,5 Prozent 1970 und 76,8 Prozent 1980 erhöht (Puwein, 1985). Die Anteile anderer Arten des Personenverkehrs gingen 
zurück: der Eisenbahnen von 27,7 Prozent 1960 auf 15 Prozent 1970 und 11,9 Prozent 1980, der Bus-Überland-Linien von 6,5 Prozent 1960 auf 4,1 Prozent 1970 und 5,8 Prozent 1980, jener städtischen Verkehrsbetriebe von 11,8 Prozent 1964 auf 6,4 Prozent 1970 und 5,5 Prozent 1980. Die Effekte anderer Formen der Eigenleistungen im Verkehr, wie die Abschaffung der Schaffner im innerstädtischen Verkehr, Ersetzung der Träger an den Bahnhöfen oder Flughäfen durch „KuliWagen“, Beförderung kleiner Lasten in gemieteten Lastautos, Fliegen mit eigenen Flugzeugen oder die Wiederbelebung des Fahrrads als Verkehrsmittel, waren weniger bedeutend.

- Tourismus: Erholungsreisen ins eigene Wochenendhaus, ins gemietete Appartement und Urlaub am Campingplatz sind die „Do-ityourself"-Formen des Tourismus: ein Teil der Leistungen der Beherbergungsbetriebe wird von den Reisenden übernommen. Zwischen 1975 und 1981 nahm die erste Form um 176 Prozent, die zweite um 195 Prozent und die dritte um 47 Prozent zu, der Anstieg aller drei betrug 147 Prozent; die gesamten Inlandsreisen in Österreich stiegen im gleichen Zeitraum nur um etwa 12 Prozent. Der Anteil der „Do-ityourself"-Reisen an den Inlandsreisen stieg zwischen 1975 und 1981 von 7,3 Prozent auf 16,2 Prozent (Bobretzky, 1984). Die Infrastruktur für die Eigenleistungen der Touristen bildeten der hohe Motorisierungsgrad und die zunehmende Zahl der Zweitwohnplätze. 1981 gab es in Österreich 161.200 Wochenend- und Ferienhäuser. Ihr Anteil am gesamten Gebäudebestand war hoch im Osten des Landes (14,8 Prozent in Niederösterreich, 14,2 Prozent im Burgenland, 13,3 Prozent in Wien); in den südlichen und westlichen Bundesländern war er niedriger und betrug zwischen 6 Prozent und 7,9 Prozent (Klein, 1986).

- Handel: Die Kunden haben, vor allem im Lebensmitteleinzelhandel, die Bedienung in den Geschäften und die Lagerung der Waren teilweise übernommen. Die Zahl der Selbstbedienungsgeschäfte im organisierten Lebensmitteleinzelhandel stieg von 34 Prozent 1967 auf 70 Prozent 1977 (ÖIV, 1978) und auf 83 Prozent 1982 (ÖIV, 1984). Der Bestand an Kühlschränken pro 1000 Personen betrug 7 Stück 1954, 265 Stück 1974 und 308 Stück 1979 (Wüger, 1981). 1979 hatten 86 Prozent, 198496 Prozent aller Haushalte einen Kühlschrank; die entsprechenden Zahlen für Gefriertruhen oder -schränke lauteten 42 Prozent 1979 und 56 Prozent 1984 (Vocelka-Zeidler, 1985).

- Unterhaltung: Die Unterhaltung durch Theaterstücke, Filme und Musik in den Haushalten - außerhalb von Theatern, Kinos und Konzertsälen - nahm stark zu. Die Zahl der Fernsehgeräte pro 1000 Personen stieg von 11 Geräten 1954 auf 242 Geräte 1974 und 279 Geräte 1979 (Wüger, 1981); 1974 hatten 80 Prozent, 197983 Prozent und 198497 Prozent der Haushalte ein Fernsehgerät (Vocelka-Zeidler, 1985). Die Zahl der Kinos und der Kinobesucher ging zurück; das Kinosterben setzte mit dem Vormarsch des Fernsehens Ende der sechziger, Anfang der siebziger Jahre ein. Vergleichbare Zahlen für eine längere Periode sind für Wien vorhanden: 1964 besuchten 25,3 
Millionen Menschen 194 Kinos, 1976 kamen 6,2 Millionen Besucher in 82 Kinos, 1984 5,9 Millionen Personen in 105 Kinos (ÖStZ, 1965, 1977, 1985). Die finanzielle Krise des Theaters hat sich vertieft, die Subventionen nahmen zu. Die Bundestheater erhielten 1970381 Millionen Schilling, 19841415 Millionen Schilling an Subventionen; direkte Subventionen für private Theater betrugen 197020 Millionen Schilling, 1984122 Millionen Schilling. Indirekt, durch Zweckzuschüsse des Bundes an Länder und Gemeinden, wurden den Theatern 1970 weitere 25 Millionen Schilling, 1984 weitere 100 Millionen Schilling zugeführt. Insgesamt betrugen die Subventionen der Theater 1970426 Millionen Schilling, 19841637 Millionen Schilling, sie sind binnen 14 Jahren um 284 Prozent gestiegen. (Der durchschnittliche Preisanstieg zwischen 1970 und 1984 betrug 96 Prozent; wird die nominelle Wachstumsrate mit diesem Wert deflationiert, betrug der „reale“ Zuwachs der Theatersubventionen zwischen 1970 und $1984+96$ Prozent. Das Brutto-Inlandsprodukt ist im gleichen Zeitraum real nur um 51 Prozent gestiegen.)

- Haushaltsarbeit: 1981 hat in Österreich eine erwachsene Person (ab 19 Jahren) im Durchschnitt (d. h. einschließlich Sonntagen und Feiertagen) ähnlich wie in anderen Industriestaaten gleich viel Zeit mit produktiver erwerbstätiger Arbeit und produktiver Arbeit für den Eigenbedarf verbracht (Simhandl, 1984). Vom gesamten Zeitaufwand für den Eigenbedarf entfielen etwa 79 Prozent auf traditionelle Haushaltsarbeit, etwa 11 Prozent auf handwerklichen Tätigkeiten und etwa 10 Prozent auf Gartenarbeit. Die Haushalte haben kräftig in die Beschaffung von Haushaltsgeräten investiert. Die Ausstattung mit Waschmaschinen je 1000 Personen stieg von 3 Stück 1954 auf 144 Stück 1974 und auf 167 Stück 1979 (Wüger, 1981). 1974 standen 64 Prozent, 197967 Prozent und 198480 Prozent der Haushalte eine Waschmaschine zur Verfügung. 1974 besaßen 4 Prozent, 197910 Prozent und 198418 Prozent der Haushalte einen Geschirrspüler. 1974 hatten 75 Prozent der Haushalte einen Staubsauger, 1979 waren es 85 Prozent, 198489 Prozent. Die Produktivität der Haushaltsarbeit stieg kräftig; viele Hausfrauen konnten einen Beruf ausüben und gleichzeitig auf bezahlte Hilfe verzichten. Die Erwerbsquote der Frauen zwischen 30 und 40 bzw. zwischen 40 und 50 Jahren $^{5}$ betrug 1964 53,3 Prozent in der ersten und 51,2 Prozent in der zweiten Altersgruppe, 1976 58,5 Prozent und 56,9 Prozent, 1984 62,4 Prozent und 60,3 Prozent. Die Zahl der Beschäftigten in der Haushaltung (Haushälterinnen, Dienstmädchen u. a.) ging von 16.439 Personen 1964 auf 11.023 Pesonen 1976 und 7.228 Personen 1984 zurück. Die Wertschöpfung (zu konstanten Preisen 1976) der „Häuslichen Dienste“ betrug 19641489 Millionen Schilling, 1976645 Millionen Schilling und 1984423 Millionen Schilling, die jährliche durchschnittliche Veränderungsrate betrug $-6,1$ Prozent.

- Eigenheimbau: Eigenleistungen und Schattenwirtschaft sind im Eigenheimbau und bei den Haus- und Wohnungsreparaturen eng verflochten. Besonders in den ländlichen Gebieten wird das Haus 
vom Eigentümer, seiner Familie und Bauarbeitern, die ihr reguläres Einkommen durch Schwarzarbeit verbessern wollen, gebaut ${ }^{6}$. Schwarzarbeit und Eigenleistungen (einschließlich Nachbarschaftshilfe) machen etwa 40 Prozent des Gesamtarbeitsaufwands aus; das Verhältnis der Schwarzarbeit zu Eigenleistung beträgt ungefähr 3:2. Der Anteil der Schwarzarbeit und der Eigenleistungen ist in einzelnen Bauphasen unterschiedlich: hoch (60 Prozent bis 70 Prozent) beim Keller- und Rohbau, hingegen niedrig (20 Prozent bis 30 Prozent) beim weiteren Ausbau der Häuser und bei den Heizungs-, Elektrizitäts- und Wasserinstallationen (Neubauer, 1984).

- Landwirtschaft: Die traditionelle landwirtschaftliche Eigenproduktion ging - in Fortsetzung des säkulären Trends - zurück. Der Anteil der Erzeugung landwirtschaftlicher Waren für den Bedarf der bäuerlichen Haushalte am Brutto-Produktionswert der Land- und Forstwirtschaft sank von 11,4 Prozent 1970 auf 6,6 Prozent 1980 - stärker in der pflanzlichen Produktion, schwächer in der tierischen Produktion; in der forstwirtschaftlichen Produktion blieb der Anteil mit etwa 3 Prozent stabil. Landwirte widmeten hingegen den Maschinenreparaturen, dem Wohnungsbau und dem Bau landwirtschaftlicher Gebäude mehr Zeit. Der Anteil der Eigenleistungen an den Bauinvestitionen der Landwirtschaft stieg von weniger als 5 Prozent Mitte der sechziger Jahre auf etwa 20 Prozent Anfang der achtziger Jahre (Schneider, 1984).

\section{Wirtschaftliche und technologische Gründe der Zunahme der Eigenleistungen}

Eigenleistungen, welche die auf dem Markt erhältlichen Dienstleistungen bzw. Waren ersetzen, haben wirtschaftliche Vorteile für die Haushalte; der technologische Fortschritt verstärkt diese Vorteile, erleichtert die Eigenleistungen oder transformiert sie (Skolka, 1976a, b, 1977). Die beiden Faktoren unterstützen einander gegenseitig: wirtschaftliche Vorteile stimulieren die Suche nach neuen Technologien, neue Technologien steigern die wirtschaftlichen Vorteile der Eigenleistungen.

Viele Dienstleistungen, einige Waren und manche Tätigkeiten in der Warenproduktion (z. B. Montage der Fertigprodukte) gehören zu den innovationsarmen (rückständigen) Tätigkeiten in der Wirtschaft; sie weisen aus technologischen Gründen einen schwachen Anstieg der Arbeitsproduktivität aus. Hohe Arbeitsintensität dieser Tätigkeiten verursacht in einem Industrieland (mit hohem Pro-Kopf-Einkommen) hohe Lohnkosten, an die hohe Sozialversicherungsbeiträge, Gemeinkosten und Steuern gekoppelt sind. Die arbeitsintensiven Waren und Dienste verteuern sich deswegen relativ?. Unter diesen Umständen kommt ihre Erbringung bzw. Herstellung im Haushalt den Konsumenten billiger: Lohnkosten, Gemeinkosten (und auch Verteilungskosten - Frachten, Werbung, Handelsspannen) die in der marktmäßigen 
Produktion verrechnet werden, fallen weg; Eigenleistungen erlauben legale Steuervermeidung (Skolka, 1976a; Scharpf, 1985). Die Konsumenten müssen arbeiten, doch das imputierte (zugerechnete) Einkommen aus dieser Eigenarbeit ist hoch. Steuern, Abgaben und Gemeinkosten haben zur Folge, daß das Pro-Stunde-Nettoeinkommen der Konsumenten aus ihrer Erwerbstätigkeit (Einkommen nach der Bezahlung von Steuern und Abgaben) nicht selten niedriger ist als der Pro-StundeBruttolohn der Beschäftigten in Dienstleistungs-, Reparatur- oder Gewerbebetrieben (der den Konsumenten verrechnet wird). Die Einkommensunterschiede in den Industrieländern, teils als Folge der Bildung breiter Bevölkerungsschichten, teils als Folge der Einkommenstransfers, sind geringer und die Belastung der Einkommen mit der Lohnsteuer und den Sozialversicherungsbeiträgen höher geworden. Bei solchen Einkommensrelationen ist es wichtig, daß die Arbeitsproduktivität im Haushalt nahe der Arbeitsproduktivität des Gewerbebetriebs, der die gleiche Leistung oder die gleiche Ware für den Markt erzeugt, liegt. Diese Voraussetzung ist oft erfüllt. In den traditionellen persönlichen Diensten (in der Betreuung von Kindern, von Kranken oder von Alten) bestehen zwischen Haushalten, privaten oder öffentlichen Organisationen kleine Produktivitätsunterschiede. In den ,,modernen" Eigenleistungen wird die Produktivität der Haushalte durch ihre „Industrialisierung“ (Huber, 1984; Joerges, 1985) gesteigert. Nach dem Zweiten Weltkrieg hat die Industrie Haushalte mit massenproduzierten Geräten, Maschinen, Verkehrsmitteln, halbfertigen Produkten und Hilfsmaterial reichlich versorgt.

Einige Haushaltsgeräte sind ein Glied in der Kette des Wandels der Dienstleistungen in Waren, mit denen wieder Dienstleistungen erbracht werden. Ein solcher Wandel kommt in den „mentalen“ (informierenden und emotionalisierenden) Dienstleistungen, die sich „,dem Geist oder der Seele zuwenden“ (Scharpf, 1985), häufig vor. Er ist nicht neu: Mit dem Niederschreiben des Homerischen Epos wurde eine Dienstleistung (Erzählung) in ein Gut (Buch) transformiert; die Erfindung des Buchdrucks ermöglichte eine Massenproduktion von Büchern. Heutehat ein ähnlicher Wandel von Theaterspielen und Musik ein bis dahin nie dagewesenes Ausmaß erreicht; arbeitsintensive professionelle künstlerische Leistungen für reiche Schichten werden in billige Massenware verwandelt. (Die Kehrseite solcher Verbreitung der Kunst ist leider oft ein Niveaurückgang ${ }^{8}$ der Unterhaltung - Tullock, 1974.) Technischer Fortschritt, relative Preise und Einkommensverteilung wirken dabei zusammen. Im Theater gab es früher große Einkommensunterschiede zwischen Zuschauern und professionellen Schauspielern, die sich mit dem wirtschaftlichen Aufschwung verminderten. Dadurch verschlechterten sich die Tauschverhältnisse zwischen den Zuschauern und den Darstellern und der wirtschaftliche Ertrag des Theaters ${ }^{9}$ ging zurück. Industrielle Innovationen boten einen billigen Ersatz der teuren Theaterunterhaltung an. Die erste, chemische und mechanische Innovationswelle schuf eine kapitalintensive Filmindustrie; die materialisierte dramatische Kunst wurde als billiges Massenprodukt in Kinos verkauft. 
Die zweite, elektronische Innovationswelle verlagerte den Konsum der Unterhaltung in die Haushalte, die in die Beschaffung der Ausrüstung investieren mußten. Das Fernsehen liefert die Unterhaltung (und die Nachrichten) in das Wohnzimmer; Filme, Theaterspiele und Fernsehprogramme werden auf Videobändern gespeichert und im Selbstbedienungsverfahren konsumiert. Die Musik hat einen ähnlichen Wandel durchgemacht: Von den großen Orchestern der feudalen Herrscher und der Kirche führt der Weg zu Eigenleistungen mit Rundfunk- und Tonbandgeräten und Plattenspielern.

Mit der Verbreitung der elektronischen Datenverarbeitung werden sich Eigenleistungen der Haushalte verstärken und in neue Bereiche wie Bildung, Gesundheitswesen, Bankwesen - (Presvelou, 1986). Ein gutes Beispiel eines funktionierenden Systems der universitären Selbstbildung ${ }^{10}$ ist die britische Fernuniversität (Open University Gershuny und Miles, 1983). Im traditionellen Hochschulunterricht hat die Verwendung von Fernsehen und von Videobändern begonnen, der Einsatz von Lerngeräten auf verschiedenen Bildungsstufen steht bevor. Einige medizinische Diagnostikgeräte und Diagnostikmittel werden auch im Haushalt verwendbar sein. Handel und Banken bereiten sich auf den Anschluß der Heimcomputer der Kunden vor.

\section{Vielseitige Motivation für Eigenleistungen}

Die Renaissance der Eigenleistungen hat allerdings nicht nur ökonomische Ursachen (die die Eigenleistungen für die Haushalte wirtschaftlich interessant machen, oder technologische Ursachen, die die Eigenleistungen durchführbar machen). Die Ursachen sind vielfältiger, wie an dem folgenden Beispiel des Wäschewaschens gezeigt wird. Das traditionelle Wäschewaschen war eine der schwersten Haushaltsarbeiten, sie wurde von Hausfrauen, als Eigenleistung, oder von bezahltem Personal, als marktmäßige Dienstleistung, erledigt. Dies hat sich in der Nachkriegszeit grundsätzlich verändert. Auskunft darüber geben u. a. französische (Préel, 1986) und schwedische (Cronberg und Sangregorio, 1982) Studien.

Im Vordergrund der französischen Studien stehen die wirtschaftlichen Motive des Wandels von den früheren Formen des Wäschewaschens - entweder in kommerziellen Wäschereien oder mit bezahltem Haushaltspersonal - zur Erledigung dieser Arbeit in kleineren automatischen Waschmaschinen in den Haushalten. Dieser Wandel verlief in Frankreich auffallend rasch: Der Anteil der Ausgaben im Haushaltsbudget für „Wäschewaschen und Reinigung“ betrug 1955 0,54 Prozent, 1970 0,37 Prozent und 1983 0,20 Prozent. Der Anteil der Ausgaben für das Wäschewaschen zu Hause stieg von 0,08 Prozent 1955 auf 0,25 Prozent 1970 und ging 1983 leicht auf 0,22 Prozent zurück. Der Anteil der Ausgaben für „Häusliche Dienste“ (Haushaltspersonal) ging von 2,21 Prozent 1955 auf 0,98 Prozent 1970 bis auf 0,48 Prozent 1983 zurück. In relativen Zahlen bei einem Indexwert von 100 für das Volumen der 
Haushaltsausgaben und das Preisniveau 1959 betrug 1984 das Volumen der Ausgaben für das Wäschewaschen in den Haushalten 10,3, das Preisniveau 138,5; das Volumen der Ausgaben für die kommerzielle Erledigung von „Wäschewaschen und Reinigung“ 39,9, das Preisniveau 123,1; das Volumen der Ausgaben für „Häusliche Dienste“ 24,3, das Preisniveau 315,1. Die relativen Preise beeinflußten die Konsumstruktur - und auch den Arbeitsmarkt. Die Zahl der Beschäftigten in den Wäschereien und chemischen Putzereien ging von 69.840 Personen 1962 auf 47.200 Personen 1982 zurück (die durchschnittliche jährliche Veränderungsrate betrug -1,94 Prozent). Dafür wird zu Hause mehr gearbeitet: der Arbeitsaufwand für das Wäschewaschen in Haushalten wird in Frankreich gegenwärtig auf 2,3 Millionen voll beschäftigter Personen geschätzt. Die relativen Preise - und mit ihnen die Nachfragestruktur haben sich in der Nachkriegszeit deutlich verändert; das Wäschewaschen zu Hause ist bequemer geworden. Es ist gegenwärtig allerdings nicht billiger als in den kommerziellen Wäschereien, die Kosten der beiden Formen sind ungefähr gleich. (Etwa 45 Prozent der Kosten des Wäschewaschens im Haushalt sind zugerechnete - imputierte - Arbeitskosten, die gleichzeitig zugerechnetes Einkommen der Haushalte fiktives Entgelt für den Arbeitsaufwand - sind.)

Die schwedischen Studien über das Wäschewaschen befassen sich mit verschiedenen Formen der Eigenleistungen der Haushalte. Um die schwere Arbeit des Wäschewaschens zu erleichtern, hat 1941 die schwedische „Population Commission“ empfohlen, mit Waschmaschinen ausgerüstete Zentralwäschereien zu errichten, in denen Hausfrauen die Wäsche entweder selbst oder mit Hilfe des Personals waschen können. Acht Jahre später mußte die Kommission feststellen, daß 70 Prozent des Wäschewaschens in Schweden noch immer händisch zu Hause durchgeführt wurde. Die Zentralwäschereien waren auf das Waschen großer Mengen stark verschmutzter Wäsche in größeren Zeitabständen eingerichtet, was den Bedürfnissen der Haushalte offensichtlich nicht entsprach. Daraufhin wurden die Wäschereien mit kleineren Maschinen ausgerüstet, aber 1973 stellte die Kommission fest, daß die Zentralwäschereien weiterhin wenig gefragt waren. Sie waren weit von den Wohnungen entfernt, die Familien mußten auf Termine warten und konnten die Wäscherei nur für begrenzte Zeit in Anspruch nehmen. Nach dem Krieg waren kleine Waschmaschinen für Haushalte auf den Markt gekommen; 1950 wurde geschätzt, daß mit ihnen 12 Prozent des Wäschewaschens erledigt wurde (verglichen mit 8 Prozent in den Waschküchen der Wohnhäuser und 2 Prozent in den zentralen Wäschereien). In neuen Häusern wurden mit kleinen Waschmaschinen eingerichtete Waschküchen gebaut, 1955 standen solche Waschküchen 12 Prozent der Haushalte, 197488 Prozent der Haushalte zur Verfügung. Viele Haushalte machten von dieser Einrichtung allerdings keinen Gebrauch; 1974 hatten 47 Prozent der Haushalte eine eigene Waschmaschine.

Die Präferenz der Haushalte für Eigenleistungen kann mit ökonomischen Faktoren allein nicht erklärt werden. In Frankreich gab es 1979 
zwischen den Kosten der Eigenleistung (wenn eigene Arbeit bewertet wird) und den Preisen der kommerziellen Wäschereien keine wesentlichen Unterschiede. Die schwedischen Schätzungen der Kosten verschiedener Formen der Eigenleistung beim Wäschewaschen für 1968 und 1969 zeigten, daß die Verwendung eigener Waschmaschinen in der Wohnung die teuerste Methode des Wäschewaschens war; die Kosten des Waschens in einer Waschküche einer Wohnanlage waren niedriger ${ }^{11}$. Die wirtschaftlichen Vorteile der Eigenleistungen im Haushalt sind nicht die einzige Ursache der Renaissance der unentgeltlichen Haushaltsarbeit. Die Konsumenten orientieren sich - oft nur intuitiv, ohne genauere Kalkulation - an einer Kosten-Nutzen-Rechnung, in der sie auch die folgenden qualitativen, nicht-monetären Aspekte berücksichtigen $^{12}$ :

- Zugang zu technologischem Wissen.

- Zeitersparnissse und Zeitsouveränität.

- Vertrauen in eigene Leistung (informationelle Asymmetrie).

- Individuelle Unabhängigkeit.

- Soziale Gleichheit.

Bei der Wahl zwischen verschiedenen Möglichkeiten des Wäschewaschens wirken alle fünf Motive. In automatischen Waschmaschinen und in Waschmitteln ist das Wissen über das Wäschewaschen verkörpert und steht den Konsumenten zur Verfügung. Konsumenten können ihre Zeit flexibler gestalten und gewinnen an Zeitsouveränität. Sie wissen, daß ihre Wäsche richtig, schonend behandelt wurde und mit fremder Wäsche nicht in Berührung kam. Die Haushalte sind vom Markt unabhängig, und sie sind alle gleich. Solche Motive wirken - neben den wirtschaftlichen und technologischen - auch in vielen am Anfang des vorliegenden Aufsatzes erwähnten Fällen der Renaissance der Eigenleistungen in Österreich: im Individualverkehr, im „Do-it-yourself-Tourismus“, im Selbstbedienungshandel, in der Unterhaltung.

\section{Zugang zu technologischem Wissen}

Wissen ist strukturierte Information, die für die Lösung gegebener Fragen relevant ist. Für die Wahl zwischen Marktproduktion und Eigenleistungen ist vorwiegend das ökonomische und technologische Wissen relevant. Ökonomisches Wissen ist das Wissen über die Märkte (über die Preise, über die Löhne, über die Kosten alternativer Herstellungstechnologien, über die Kosten und die Verfügbarkeit von Produktionsfaktoren). Technologisches Wissen ist das Wissen über die und die Erbringung von Dienstleistungen. Beides Wissen wird entweder durch Erfahrungen in der Produktion oder durch systematische wissenschaftliche Forschung geschaffen. Es wird im menschlichen Gedächtnis gespeichert oder symbolisch - schriftlich, heute auch elektronisch - registriert. Das technologische Wissen wird weiters in Werkzeugen, Geräten, Maschinen, Transportmitteln, Bauten, modernen Rohstoffen und vielen Verbrauchsgütern verkörpert. 
Der Wandel von der traditionellen Gesellschaft in eine industrielle (der vor zwei Jahrhunderten begonnen hat) und dessen Fortsetzung, der Wandel in eine Informationsgesellschaft (der heute beginnt) wird durch den Wandel in der Schaffung, Speicherung, Verkörperung und Verbreitung des Wissens begleitet und gefördert (Skolka, 1987). In der traditionellen vorindustriellen Gesellschaft wurde einfaches Wissen verwendet, das durch Erfahrungen erworben und innerhalb von Familien, in Traditionen und Bräuchen von Generation an Generation weitergegeben wurde. In der traditionellen Produktion beherrschten einzelne Menschen einen großen Teil des verfügbaren Wissens und waren auf das Wissen anderer wenig angewiesen. In der Industriegesellschaft werden in der Produktion neben dem praktischen Wissen auch Erkenntnisse der wissenschaftlichen Forschung angewendet. Das Wissen wird umfangreicher, wird unter spezialisierten Fachleuten verteilt, in Fachliteratur beschrieben und in spezialisierten Maschinen, Einrichtungen und Verkehrsmitteln verkörpert. Im Bildungssystem wird es von einer Generation an die nächste übergeben. Die Verteilung des Wissens unter einer großen Anzahl von Menschen erfordert intensive Arbeitsteilungen in der Produktion und im sozialen Leben. Die elektronische informationsverarbeitende Technologie (die als „Epizentrum der gegenwärtigen technologischen Schockwelle“ bezeichnet wird) eröffnet gegenwärtig neue Möglichkeiten der Speicherung, Verkörperung und Verbreitung (Übergabe) des Wissens. Die hohe „Wissensintensität“ der elektronischen Einrichtungen (Computer, Roboter usw.) steht ihren Benützern zur Verfügung und macht sie vom Wissen anderer Menschen weniger abhängig.

Dieselben technologischen Veränderungen setzten sich, mit gewisser Verspätung, in der Haushaltsproduktion durch. Die traditionelle Haushaltsarbeit hatte einfache Technologie verwendet. In den letzten Jahrzehnten wurden Haushalte mit Investitionen in langlebige Güter ,industrialisiert". Der Einsatz von elektronischen Einrichtungen, der bevorsteht, wird die Haushaltsarbeit in die Informationsgesellschaft integrieren. Die Öffnung des Zugangs zu technologischem Wissen für die Haushalte - sei es durch Haushaltsmaschinen, sei es durch elektronische Einrichtungen - fördert schon heute die Eigenleistungen. Damit verlieren einige Arbeitsteilungen, die in der Industriegesellschaft entstanden sind, an Bedeutung: Ein Teil der produktiven Arbeit verlagert sich vom Markt zurück in die Haushalte; monetäre Kriterien werden hie und da durch Nutzwerte verdrängt. Enge Spezialisierung wird in der erwerbstätigen Arbeit nicht so hoch wie früher geschätzt, verlangt werden eher multiprofessionelle Arbeitskräfte mit allgemeinen Kenntnissen (Kern und Schumann, 1984). Dasselbe gilt auch für Haushalte, Hausfrauen (und Hausmänner) müssen heute imstande sein, mit modernen Haushaltseinrichtungen vieles zu erledigen, was früher wegen des Mangels an Fachwissen nicht möglich war. 


\section{Zeitersparnisse und Zeitsouveränität}

In jeder Gesellschaft teilen Menschen die verfügbare Zeit in biologisch notwendige (Schlaf, Körperpflege usw.), produktive und konsumptive Tätigkeiten auf. Die waren in der vorindustriellen traditionellen Gesellschaft nicht streng voneinander getrennt, eher in einem „Lebensstil“" (Usher, 1980) verschmolzen. Während der Industrialisierung trennte sich die Erwerbsarbeit scharf von der ,übrigen“ Beschäftigung (teils Freizeit ${ }^{13}$, teils Eigenleistungen). Zur Koordinierung der Arbeitsteilungen wurde eine strenge Regelung der Zeitverwendung eingeführt: „Die Uhr, nicht die Dampfmaschine, ist die Schlüsselmaschine des modernen Industriezeitalters" (Mumford, 1934 ${ }^{14}$ ).

Die Fixierung des Zeitablaufs der erwerbstätigen Arbeit in der Industriegesellschaft beschränkt die Zeitsouveränität der Menschen: die Länge der Erwerbsarbeit - wöchentliche Arbeitszeit, Öffnungszeiten der Geschäfte und Büros, Ordinationsstunden -, die Länge des Urlaubs-, der Schulferien, das Pensionsalter sind gesetzlich geregelt. Sie werden dem Produktivitätsfortschritt angepa $3 \mathrm{t}^{15}$. Die erwerbstätige Arbeit wurde verkürzt, aber die Menschen klagen über zunehmende Zeitknappheit (Burestam-Linder, 1970). Sie sind in der durch die Verkürzung der erwerbstätigen Arbeit gewonnenen Zeit mit neuen Aufgaben beschäftigt. Die Zeit, welche Haushalten für den Konsum der erzeugten Waren und Dienste zur Verfügung steht, hat weniger zugenommen als das reale Volumen des Konsums, der Pro-Stunde-Konsum ist gestiegen ${ }^{16}$. Zeitaufwendiger Konsum wird verdrängt $t^{17}$, auch auf Kosten der Lebensqualität (Oates und Baumol, 1972; Tullock, 1974). Auch die Bewältigung komplizierter zwischenmenschlicher und zwischeninstitutioneller Beziehungen ${ }^{18}$, die durch die intensiven Arbeitsteilungen verursacht werden, nimmt immer mehr Zeit in Anspruch. Der populäre Slogan „time is money“ ist nicht symmetrisch (bzw. als mathematische Gleichung nicht kommunitativ), Zeit kann mit Geld nicht direkt gekauft werden. Sie kann aber mit Hilfe von Dienstleistungen (entweder der marktmäßigen oder der unentgeltlichen, öffentlichen) gewonnen werden. Der zeitsparende Effekt der Dienstleistungen zeichnet sich auch in ökonometrischen Konsumfunktionen ab. Die positiven Einkommenselastizitäten für die warenintensiven Aufwandsarten sind kleiner und für die dienstleistungsintensiven Aufwandsarten größer als eins; die negativen Preiselastizitäten für die dienstleistungsintensiven Aufwandsarten sind höher als jene für die warenintensiven ${ }^{19}$. Die hohen Einkommenselastizitäten der dienstleistungsintensiven Aufwandsarten könnten auch als Zeichen des zunehmenden Zeitmangels, die hohen negativen Preiselastizitäten als Zeichen höherer Bewertung der Zeit bei steigendem Einkommen verstanden werden. Die langfristig fast stabilen relativen Unterschiede ${ }^{20}$ im marktmäßigen Konsum von Waren und Dienstleistungen zwischen verschiedenen Einkommensklassen sind durch diese Komplementarität der Einkommens- und Preiselastizitäten weitgehend bestimmt.

Dennoch werden die marktmäßigen Dienstleistungen - trotz ihres 
zeitsparenden Effekts - durch Eigenleistungen ersetzt, welche die Zeit des Konsumenten beanspruchen. Eine Ursache dafür sind die schnell steigenden relativen Preise der Dienstleistungen (die überwiegend dem innovationsarmen, technologisch rückständigen Sektor angehören). Ein anderer, bisher wenig beachteter Grund dieser Substitution ist, daß Eigenleistungen die Zeit wirksamer als marktmäßige Dienstleistungen sparen können. Die Erklärung dieses Paradoxons liegt im unregelmäßigen Zeitverlauf der Nachfrage. Dienstleistungen können nicht auf Lager erzeugt werden; den Betrieben entstehen durch das Warten auf Kunden Verluste; in den Stoßzeiten müssen die Konsumenten auf Bedienung warten. Eigenleistungen werden hingegen in dem Moment erbracht, in dem sie benötigt werden; weder der Produzent noch der Konsument müssen warten, da die beiden in derselben Person verkörpert sind. Das tägliche Leben zeigt dies anschaulich: Männer rasieren sich am Morgen, Frauen pflegen ihre Frisur; Menschen fahren an ihren Arbeitsplatz, gehen mittags essen und erledigen am Abend Einkäufe. Alle könnten die dazu betreffenden marktmäßigen Dienstleistungen in Anspruch nehmen: beim Friseur, in den öffentlichen Verkehrsmitteln, in Restaurants und Geschäften mit Bedienung. Sollten sich aber alle Konsumenten so verhalten, wäre der Bedarf nach Arbeitskraft und Ausrüstung in diesen Diensten in den Stoßzeiten enorm, und den Kunden würden längere Wartezeiten nicht erspart bleiben. Theoretisch könnten Mehrzweckarbeitskräfte ausgebildet werden, die den Arbeitstag als Friseure beginnen und sich später als Straßenbahnlenker und Schaffner, Kellner und Verkäufer betätigen würden. (Eine ähnlich vielseitig verwendbare Ausrüstung ist noch schwerer vorstellbar.) Die weitaus effizientere Lösung besteht allerdings darin, daß sich die Konsumenten in Mehrzweckkräfte verwandeln. Sie rasieren und frisieren sich selbst, chauffieren ihren eigenen PKW, essen in Selbstbedienungskantinen und kaufen in Selbstbedienungsgeschäften ein. Die Eigenleistungen beanspruchen zwar Zeit und Arbeitsaufwand der Konsumenten, aber sie ersparen ihnen das Warten und erhöhen die Flexibilität der Zeitverwendung.

\section{Vertrauen in eigene Arbeit (Informationsasymmetrie)}

Die meisten Eigenleistungen der Haushalte sind Dienstleistungen, Waren werden in Haushalten seltener erzeugt. Dienstleistungen und Waren unterscheiden sich durch mehrere Eigenschaften. Die eine ist die „Informationsasymmetrie“, sie kommt bei den Dienstleistungen häufiger als bei den Waren vor: Der Kunde weiß weniger über die Qualität der Leistung als der Produzent, kann sie ex ante nur schwierig oder gar nicht beurteilen. Beim Kauf einer Dienstleistung ist dieses Risiko größer als beim Kauf einer Ware. Eine mangelhafte Ware kann repariert oder gegen neue getauscht werden, eine fehlerhafte Dienstleistung verändert den Zustand des Eigentums des Kunden (einer Ware, oder seines Körpers) und ist oft irreversibel. 
Eine Form der Informationsasymmetrie ist, daß der Käufer die Qualität der angebotenen Dienstleistung schwer überprüfen kann („moral hazard“ - Stiglitz, 1983). Sie ist z. B. im Gesundheitswesen vorhanden, kommt aber auch bei einfacheren Diensten vor: im Restaurant weiß der Gast nicht, ob die Rohstoffe, die der Koch verwendet hat, frisch waren, ob die Küche sauber ist usw.; in der öffentlichen Wäscherei weiß der Kunde nicht, ob seine Wäsche in Berührung mit fremder Wäsche gekommen ist; in der Betreuung der Kinder, der Kranken, der Gebrechlichen oder der Alten hängt die Qualität der Pflege von der persönlichen Beziehung des Pflegers zum Betreuten ab. Bei einer anderen Form der Informationsasymmetrie wird das Ausmaß der benötigten Leistung beim Angebot überschätzt (,,adverse selection“). Die angebotenen unnötigen Leistungen werden entweder tatsächlich durchgeführt; oder es wird nur die notwendige Leistung durchgeführt und die angebotene überhöhte Leistung dem Kunden verrechnet. Solche Fälle kommen bei der Reparatur von Autos, Geräten und Einrichtungen vor ${ }^{21}$.

Auf die Informationsasymmetrie reagieren die Produzenten und die Konsumenten unterschiedlich. Der Produzent versucht, das Vertrauen der Kunden - den ,guten Ruf" (Reputation - Holmstrom, 1984) - zu gewinnen. Er baut mit perfekten Leistungen einen Kundenstock auf, investiert in Werbung, unterwirft sich öffentlichen Kontrollen und Prüfungen. Der Kunde kann dennoch mißtrauisch bleiben und - falls er die notwendigen Kenntnisse und Ausrüstung hat - die Leistung selbst erbringen. Eigenleistungen schließen die Informationsasymmetrie aus: Man weiß, was notwendig war und was tatsächlich geleistet wurde.

\section{Individuelle Unabhängigkeit}

Freie Zeitgestaltung - Zeitsouveränität - und die Fähigkeit, der Informationsasymmetrie durch Eigenleistung auszuweichen, sind wesentliche Aspekte individueller Unabhängigkeit. In der traditionellen Gesellschaft war die freie Zeitgestaltung durch den Ablauf der biologischen Prozesse in der Landwirtschaft und durch die soziale Ordnung beschränkt. Die Haushalte besaßen alle für die damals überwiegende Produktion für den Eigenverbrauch notwendigen Kenntnisse. Koordinierung der Arbeitsteilungen, genauere Zeitmessung und wachsende Abhängigkeit vom Wissen anderer Menschen haben im Industriezeitalter die individuelle Unabhängigkeit beschränkt. Mit der Renaissance der Eigenleistungen wird sie wieder erhöht. Eigenleistungen sind auf das Wissen anderer wenig angewiesen und können zu beliebiger Zeit getätigt werden. Auch wenn der Begriff ,Schattenwirtschaft" mit dem der Eigenleistungen nicht ganz identisch ist, gilt für diese folgendes: „Auf den einfachsten Nenner gebracht, erscheint die Schattenwirtschaft als die Summe der Versuche von Menschen, ihre Probleme selbst zu lösen“ (Groser, 1984).

Das Gefühl größerer individueller Unabhängigkeit, das die Eigenlei- 
stungen vermitteln, ist zum Teil trügerisch. Eigenleistungen können ohne Material, Energie, Werkzeuge, Maschinen, Verkehrsmittel, die nur auf dem Markt erhältlich sind, nicht getätigt werden. Die zu deren Kauf notwendigen Mittel müssen durch erwerbstätige Arbeit verdient werden. Weil moderne Eigenleistungen eng an den Markt gebunden sind, entsteht eine neue Form der Abhängigkeit. Für die traditionelle landwirtschaftliche Eigenproduktion war nur der freie Zugang zum Boden unentbehrlich; Rohstoffe, einfache Werkzeuge und Bauten wurden in Eigenregie erzeugt. Moderne Eigenleistungen schaffen die Abhängigkeit von den Arbeitsteilungen nicht ab: Unmittelbare Abhängigkeit von den Leistungen anderer Leute wird in Abhängigkeit von Ausrüstung, Material und Energie für Eigenleistungen, zu deren Einkauf monetäres Einkommen notwendig ist ${ }^{22}$, verwandelt; die Renaissance der Eigenleistungen hängt vom Zugang zur erwerbstätigen Arbeit ${ }^{23}$ ab (Archambault, 1985). Deswegen sind Eigenleistungen ein Privileg - und eine Domäne - der breiten Mittelschicht, die ein nicht niedriges und gesichertes Einkommen hat; die Armen können die Maschinen und Werkzeuge nicht kaufen, die Reichen können die teuren marktmäßigen Dienste bezahlen und auf Eigenleistungen verzichten.

In diesem Punkt berührt die Problematik der Eigenleistungen die Problematik des ,,arbeitslosen Grundeinkommens“, das bei der angeblich drohenden permanenten technologischen Arbeitslosigkeit (Leontief, 1983) die Grundbedürfnisse aller Bürger sichern soll. Der Staat solle dieses Einkommen durch Umverteilung eines Teiles des Nationaleinkommens sichern. Solche Ideen - deren Vorbilder in utopischen Schriften der Vergangenheit zu finden sind - wurden in der letzten Zeit in Westeuropa, vorwiegend im deutschsprachigen Raum, geäußert (Gretschmann, 1985; Schaff, 1985; Opielka und Votruba, 1986); in den Vereinigten Staaten tauchten sie schon früher auf (Brüder Goodman, 1968; Theobald, 1965). In der amerikanischen Diskussion wurde vor kurzem vorgeschlagen, daß die Grundbedürfnisse der Bürger mit einer Vermögensumverteilung statt einer Einkommensumverteilung gesichert werden sollen (Davidson, 1985; Morehouse, 1985; Speiser, 1985). Die Fragen, ob das arbeitslose Grundeinkommen eine ausreichende Einkommensquelle für die Beschaffung der Ausrüstung und des Materials für Eigenleistungen sein könnte, ob Eigenleistungen zur Milderung der technologischen Arbeitslosigkeit geeignet wären ${ }^{24}$ und ob die Umverteilung des Einkommens oder des Vermögens die individuelle Unabhängigkeit erhöhen oder vermindern würde, wurden bisher nicht berührt.

\section{Soziale Gleichheit}

Die Idee sozialer und ökonomischer Gleichheit ist Bestandteil vieler Religionen und sozialer Bewegungen, man findet sie auch bei den ersten Ökonomen vor Adam Smith. In der Nachkriegszeit haben die industrialisierten Länder eine Verminderung der Einkommensunter- 
schiede erreicht: dank des kräftigen Wirtschaftswachstums, dank der Verbreitung der Bildung, dank der keynesianischen Wirtschaftspolitik ${ }^{25}$ und dank der Einkommenstransfers und Leistungen des Wohlfahrtstaates (Paukert, 1973). Unbemerkt blieb, daß die Renaissance der Eigenleistungen auch zum Ausgleich der Vermögensverteilung und der Belastung durch Haushaltsarbeit beigetragen hat. Dies wird mit drei Beispielen belegt; die ersten zwei betreffen die Investitionen der Haushalte, das dritte die Haushaltsarbeit.

Die Investitionen der Haushalte in Haushaltseinrichtungen und Verkehrsmittel (und auch in Zweitwohnungen) wuchsen in der Nachkriegszeit kräftig. Die amerikanischen Haushalte besaßen 1966 40,9 Prozent des Gesamtvermögens der Vereinigten Staaten; davon entfiel ein Viertel (11,8 Prozent) auf Maschinen und Geräte. Der Anteil der privaten Unternehmungen betrug 41,7 Prozent, davon waren 11,6 Prozent Maschinen und Einrichtungen. Die amerikanischen Haushalte hatten den Hauptanteil am Fixkapital in drei Dienstleistungsbereichen: Wohnen, Unterhaltung und Personalverkehr. Die Investitionen in dieses Kapital waren ertragreich: der zugerechnete Gewinn (imputed return on capital) pro Jahr wurde auf 10 Prozent bis 40 Prozent geschätzt (Poapst und Waters, 1964; Juster, 1966; Burns, 1977; Katz und Peskin, 1980). In der Bundesrepublik Deutschland betrug das Vermögen der privaten Haushalte (zu konstanten Preisen 1976) 1970398 Milliarden DM, 1984945 Milliarden DM; es hat binnen 14 Jahren um 137 Prozent zugenommen (Schäfer, 1985).

Eigenleistungen gleichen die Unterschiede in der Belastung durch die Haushaltsarbeit aus. Die Zahl der bezahlten Haushaltskräfte ging in der Nachkriegszeit zurück, viele Haushalte konnten sich infolge des Anstiegs der Reallöhne Haushaltskräfte nicht leisten. Sie verwandelten sich in mit Maschinen und Geräten ausgestattete SelbstbedienungsHaushalte, in denen verschiedene soziale Schichten ungefähr den gleichen Arbeitsaufwand leisten müssen. In Großbritannien verbrachten 1937 die Hausfrauen der Arbeiterklasse zweimal so viel Zeit mit der Haushaltsarbeit als Hausfrauen der Mittelklasse. 1961 waren die Arbeiterfrauen mit der Haushaltsarbeit weniger, die Frauen der Mittelklasse aber stärker zeitlich belastet als in den dreißiger Jahren. Die Unterschiede zwischen den beiden Gruppen haben sich wesentlich vermindert, aber beide Effekte zusammen ergaben - paradoxerweise - einen leichten Anstieg der durchschnittlichen Belastung aller Frauen (Gershuny, 1983). Der bevorstehende Einsatz der datenverarbeitenden Technologie in Haushalten wird vermutlich die Tendenz zur Gleicheit verstärken und besonders den Zugang zu akkumuliertem Wissen (in den Datenbanken oder in Expertensystemen) für breite Bevölkerungsschichten öffnen.

Die gegenwärtige Renaissance der Eigenleistungen hat wichtige technologische und ökonomische Gründe: unterschiedliche Produkti- 
vitätsentwicklung in der Waren- und Dienstleistungsproduktion, unterschiedliche Preisentwicklung, steigende Steuer- und Abgabenbelastung und Verminderung der Einkommensunterschiede. Diese Faktoren allein können die Renaissance der Eigenleistungen allerdings nicht erklären ${ }^{26}$. Breiter Zugang zu technologischem Wissen, Zeitersparnisse und Zeitsouveränität, Informationsasymmetrie, Präferenz für die Unabhängigkeit und soziale Gleichheit sind die weiteren Gründe für die Zunahme der Eigenleistungen.

\section{Anmerkungen}

1 Der vorliegende Artikel ist eine leicht überarbeitete Version des Beitrags zur Konferenz über Wohlfahrtsproduktion zwischen Markt, Staat und Haushalt, Zentrum für interdisziplinäre Forschung, Universität Bielefeld, 18.-20. September 1986.

2 Der Terminus „Arbeitsteilungen“ wird in Anlehnung an das Buch des englischen Ökonomen Pahl „Divisions of Labour“ (1984) verwendet.

3 Mit der Industrialisierung änderte die Wirtschaftstheorie ihre Auffassung über die produktive Arbeit. Produktiv war für F. Quesnay (1758) nur die Landwirtschaft, für A. Smith (1776) die Produktion materieller Güter. Nach der gegenwärtigen Auffassung ist jene Leistung produktiv, die Nutzen stiftet und Gegenstand freiwilliger Nachfrage ist: „It is productive because it is valued“ (Robbins, 1932). Laut M. Reid (1934) sind auch solche Eigenleistungen produktiv, ,die eine dritte Person gegen Bezahlung erbringen könnte“.

4 Vor zweihundert Jahren deckten Haushalte in Westeuropa und in Nordamerika ungefähr 75 Prozent ihres Bedarfs durch Eigenproduktion, erwarben etwa 20 Prozent durch Tausch auf den lokalen Märkten und 5 Prozent durch Geld (Burns, 1977). Der Anteil der Eigenleistung ist heute auf die Hälfte gesunken (Goldsmith/Clermont, 1982).

5 Trotz der steigenden Beschäftigung der Hausfrauen in den Städten blieb die Erwerbsquote aller Frauen im erwerbsfähigen Alter wegen der steigenden Zahl von Studentinnen und des Rückgangs an Bäuerinnen fast unverändert: zwischen 1964 und 1976 stieg sie nur von 55,2 Prozent auf 57,1 Prozent.

6 Ähnliche Angaben für die BRD bringen Jessen u. a. $(1984,1986)$ und Heinze, Schedl und Vogler/Ludwig (1986).

7 Die Relation ist auch mit dem Niveau des Pro-Kopf-Inlandsprodukts stark korreliert. Anfang der siebziger Jahre betrug das Verhältnis des Preises eines kleinen Pkw zu dem eines Haarschnitts 4000:1 in Portugal, 2400:1 in Großbritannien, 1200:1 in Schweden und 700:1 in den USA (OECD, 1974).

8 "Economic prosperity does not automatically bring with it all the things that are usually taken to contribute to the quality of life and if it is accompanied by rising real incomes for all economic classes, it may be a positive detriment to such activities" (Oates und Baumol, 1972).

9 Zwischen 1670 und 1970 ist die Produktivität eines Genfer Uhrmachers auf das 200 fache gestiegen. 1680 schrieb Purcell die Oper „Dido und Aeneas“; sie braucht heute die gleiche Zahl an Darstellern und Musikern wie vor 200 Jahren (Baumol, Batey und Wolff, 1985). Shakespeares Stücke waren zu seiner Zeit nach zwei Wochen Spielzeit kostendeckend. Vor dem Ersten Weltkrieg wurde dieser „,break-even-point“" nach vier Wochen, in den zwanziger Jahren nach fünf bis acht Wochen und Anfang der siebziger Jahre nach siebzig Wochen Spielzeit erreicht (Oates und Baumol, 1972).

10 Die Fernuniversität verwendet Lehrbücher, Skripten und Fernsehprogramme; die Studenten sind erwerbstätig und lernen zu Hause. Die Fernuniversität ist um 40 Prozent bis 60 Prozent billiger als die konventionelle Massenuniversität (Gershuny und Miles, 1983).

11 Die Kosten waren wie folgt: $1,30 \mathrm{skr}$ pro Kilogramm Wäsche bei Verwendung einer eigenen Waschmaschine in der Wohnung; in der Waschküche einer Wohnanlage 
betrugen sie $0,78 \mathrm{skr}$ pro Kilogramm (bei 25 Wohneinheiten) bzw. 0,74 skr pro Kilogramm (bei Wohneinheiten); in einer Zentralwäscherei 0,86 skr (bei 25 Wohneinheiten), $0,78 \mathrm{skr}$ (bei 105 Wohneinheiten) und $0,71 \mathrm{skr}$ (bei 210 Wohneinheiten).

12 Verschiedene Typen von Haushalten weisen auch unterschiedliche Neigungen zu Eigenleistungen auf. Capecchi und Pesce (1986) unterscheiden zwischen vier Haushaltstypen: 1) selbstversorgende (autosuffisante); 2) konsumorientierte (consomatrice); 3) individualistische (individualiste) und 4) kollektivorientierte (associative). Jeder Typ hat auch eine andere Haltung zu den marktmäßigen und öfentlichen Dienstleistungen.

13 „Freizeit ist jene Zeit, die man frei von allen Notwendigkeiten und Verpflichtungen rein zum Vergnügen oder zum Nichtstun übrig hat" (Gehmacher, 1980).

14 "The clock, not the steam engine, is the key machine of the modern industrial age" (Mumford, 1934).

15 Die wöchentliche Arbeitszeit wurde in den meisten Industrieländern von etwa 80 Stunden um 1900 auf gegenwärtig etwa 40 Stunden verkürzt, bis zum Ende des Jahrhunderts wird sie vermutlich auf etwa 35 Stunden sinken. Bezahlter Urlaub wurde von einer bis zwei Wochen Anfang des Jahrhunderts auf etwa einen Monat verlängert, bis zum Ende des Jahrhunderts kommt vermutlich noch eine Woche dazu. In Österreich wurden zwischen 1964 und 1982 etwa 20 Prozent der Steigerung der Arbeitsproduktivität nicht als höherer privater Konsum von Gütern und Dienstleistungen, sondern als Verkürzung der Arbeitszeit konsumiert (Mitter und Skolka, 1984).

16 In der Schweiz hat sich zwischen 1900 und 1980 das Volumen der „Freizeit“ verdoppelt, der Umfang der verfügbaren materiellen Güter hat sich verelffacht (Fritsch, 1984).

17 "Utility is a function not only of commodities, but also of the time allocated to them" (DeSerpa, 1971).

18 Beim Telefonieren sind es in den Entwicklungsländern die versuchten Anrufe bei anderen Personen, in den Industrieländern sind es hingegen die erhaltenen Anrufe anderer, die einen zur Verzweiflung bringen: „I tell my students that one excellent way to tell apart the underdeveloped from the developed countries is by looking at their phone systems: in the underdeveloped countries, you go crazy making telephone calls; in the developed countries, receiving them!" (Bhagwati, 1987).

$19 \mathrm{Da}$ die relativen Preise der dienstleistungsintensiven Aufwandsarten langfristig schneller als die der warenintensiven Aufwandsarten steigen, ergibt diese Konstellation der Elastizitäten beim steigenden Einkommen der Haushalte eine fast stabile reale Verteilung des Konsums auf waren- und dienstleistungsintensive Aufwandsarten. Empirische Untersuchungen bestätigen diese Stabilität (Carré, 1960; Fuchs, 1968; Summers, 1985).

20 "A poor person in an industrialized country should not be expected to behave in his consumption pattern like a person at the same income level in a developing country, and in the course of economic development the low income groups cannot hope to achieve the present consumption pattern of the high income groups" (Lancaster, 1966).

21 Die Österreichische Zeitschrift „Konsument“ hat in Nr. 8/1984, S. 7, einen Leserbrief des Herrn Carl Sehner aus Königsbrunn zum Thema „Facharbeit-Pfusch“ veröffentlicht: „Vor etwa zehn Jahren begann mein alter Schwarzweißapparat zu streiken. Guter Ton, aber kein Bild. Kommentar eines Fernsehbetriebes: Den können's wegwerfen, da ist die Bildröhre kaputt. Als Halbfachmann machte mich dies stutzig. So schrieb ich an die Erzeugerfirma und bekam prompt, nebst einem freundlichen Begleitschreiben, kostenlos einen Schaltplan zugeschickt. Ein kleiner Widerstand (damalige Kosten S 5,50) war kaputt. Nach dessen Austausch funktionierte das Gerät noch sechs Jahre anstandslos!" Herr Sehner schildert weiters drei ähnliche Fälle und schließt den Brief mit folgender Frage: „Wie viele Konsumenten werden und wurden geschädigt?"

22 Delmas und Gadrey (1987) zeigen mit einem mathematischen Modell, daß monetäres Einkommen eine Voraussetzung für die Eigenleistungen ist.

23 "Informal work depends on formal work" (Jessen u. a., 1984).

24 „Im Haushalt kann es keine technologische Arbeitslosigkeit geben“ (Macek, 1945).

25 In der Lehre von Keynes dient die Umverteilung des Einkommens von den reichen zu den ärmeren Haushalten zur Steigerung der Wirtschaftstätigkeiten und zum Erreichen 
der Vollbeschäftigung. “... it must not be forgotten that he (Keynes) rendered a decisive service to equalitarianism in an all-important point. Economists with an equalitarian bent had long before learned to discount all other aspects or functions of inequality of income except one: like J. S. Mill they had retained scruples concerning the effects of equalitarian policies upon saving. Keynes freed them from these scruples" (Schumpeter, 1954).

26 Jessen u. a. $(1984,1986)$ betonen, daß die Eigenleistungen der Haushalte mit dem klassischen Modell des „homo oeconomicus“ allein nicht erklärt werden können. Die Kriterien der „moral economy“ (Thompson, 1971) müssen auch berücksichtigt werden.

\section{Literatur}

Archambault, E. (1985): «Travail domestique et emploi tertiaire: Substitution ou complémentarité», in Vernières, M. (Hrsg.): L'Emploi du tertiaire, Economica, Paris, S. 189-205

Baumol, W. J. (1967): "Macroeconomics of Unbalanced Growth: The Anatomy of Urban Crisis", The American Economic Review, Nr. 2, S. 415-426

Baumol, W. J., Batey Blackmann, S. U., Wollf, E. N. (1985): "Unbalanced Growth Revisited: Asymptotic Stagnancy and New Evidence", The American Economic Review, Nr. 4, S. 806-817

Bhagwati, J. (1987): "International Trade in Services and its Relevance for Economic Development", in: Giarini, O., The Emerging Service Economy, Pergamon Press, Oxford, S. 3-34

Bobretzky, H. (1984): „Die Do-it-yourself-Komponente im Fremdenverkehr“, in: Skolka, J. (Hrsg.): Die andere Wirtschaft: Schwarzarbeit und Do-it-yourself in Österreich, Signum, Wien, S. 179-218

Burestam-Linder, S. (1970): The Harried Leisure Class, Columbia University Press, New York

Burns, S. (1977): The Household Economy: Its Shape, Origins and Future, Beacon Press, Boston

Cappecchi, V., Pesce, A. (1986): Services aux ménages: La situation italienne, Final Report to the FAST Programme, EEC, Brüssel

Carré, P. (1960): Etude empirique sur l'évolution des structures d'économies en état de croissance, Editions du Centre National de la Recherche Scientifique, Paris

Cronberg, T., Sangregorio, I.-L. (1982): "Within our own Walls: New Technology and its Impact on Lifestyle", in UNEP - United Nations Environment Programme: Life-Styles, Environment and Development: A European Perspective, Nairobi, S. 106-123

Delmas, B., Gadrey, J. (1987): Examen de la théorie de la substitution des biens et des services, Rencontre Internationalle sur la Dynamique des Services et Théories Economiques, Université de Lille I, Lille

Davidson, P. (1985): "Can Effective Demand and the Movement toward further Income Equality be Maintained in the Face of Robotics? An Introduction", Journal of Post Keynesian Economics, Nr. 3, S. 422--425

DeSerpa, A. C. (1971): "A Theory of the Economics of Time", The Economic Journal, Nr. 324, S. 828-846

Fritsch, B. (1984): Zukunftsgestaltung durch Innovation, Institut für Wirtschaftsforschung, Eidgenössische Technische Hochschule, Zürich

Fuchs, V. R. (1968): The service economy, Columbia University, New York

Gehmacher, E. (1980): „Das Freizeitverhalten der Österreicher“, Erwachsenenbildung in Österreich, Nr. 6, S. 398-410

Gershuny, J. I. (1983): Social Innovation and the Division of Labour, Oxford University Press, Oxford

Gershuny, J. I., Miles, I. (1983): “The New Service Economy”, Frances Pinter, London

Goldschmidt-Clermont, L. (1982): Unpaid Work in the Household, International Labour Office, Genf

Goodman, P., Goodman, P. (1960): Communitas, Vintage Books, New York

Gretschmann, K. (1985): „Garantiertes Minimum: Vom Recht auf Arbeit zum Recht auf Einkommen“, in: Brun, R. (Hrsg): Erwerb und Eigenarbeit, Fischer, Frankfurt, S. 85-99 
Groser, M. (1984), „Die Schattenwirtschaft als Herausforderung für die Wirtschafts- und Sozialpolitik“, in: Katholische Akademie Schweiz, Arbeitsgesellschaft im Wandel, Schwek, S. 16-25

Heinze, R. G., Olk, T. (1982): "Development of the Informal Economy", Futures, Nr. 2, S. $189-204$

Heinze, J., Schedl, H., Vogler-Ludwig, K. (1986): „Wachstumsfelder am Rande der of fiziellen Wirtschaft", IFO-Institut für Wirtschaftsforschung, München

Holmstrom, B. (1985): "The Provision of Services in a Market Economy", in: Inman, R. P. (Hrsg.) (1985): "Managing the Service Economy: Prospects and Problems", Cambridge University Press, Cambridge, S. 183-213

Huber, H. (1984): "Public Help and Self Help", Futures, Nr. 2, S. 139-147

Jessen, J., Siebel, W., Siebel-Rebell, C., Walther, U.-J., Weyrather, I. (1986): „Versorgungsstrategien von Arbeiterhaushalten in städtischen und ländlichen Lebenssituationen“, Universität Oldenburg, Oldenburg

Jessen, J., Siebel, W., Siebel-Rebell, C., Walther, U.-J., Weyrather, I. (1987): "The Informal Work of Industrial Workers. Present Situation, Trend Prognosis and Policy Implications", in: Alessandrini, S., Dallago, B., The Unof ficial Economy, Gower, Aldershot, S. 271-282

Joerges, B. (1985): „Eigenarbeit unter industriellen Bedingungen“, in: Brun, R. (Hrsg.): Erwerb und Eigenarbeit, Fischer, Frankfurt, S. 29-45

Juster, F. T. (1966): Household capital formation and financing 1897-1962, NBER, New York

Katz, A. J., Peskin, J. (1980): "The Value of Services Provided by the Stock of Consumer Durables, 1947-1977: An Opportunity Cost Measure", Survey of Current Business, July, S. 22-31

Kern, H., Schumann, M. (1984): Das Ende der Arbeitsteilung? Rationalisierung in der industriellen Produktion, C. H. Beck, München

Klein, K. (1986): „Wochend- und Ferienhäuser“, Statistische Nachrichten, Nr. 6, S. 471-475

Lancaster, K. J. (1966): "A New Approach to Consumer Theory", The Journal of Political Economy, Nr. 2, S. 132-157

Leontief, W. (1983): "Technological Advance, Economic Growth, and the Distribution of Income", Population and Development Review, Nr. 3, S. 403-410

Macek, J. (1945): Socialní ekonomika (Soziale Wirtschaftslehre), Česká grafická Unie, Prag Mitter, P., Skolka, J. (1984): "Labour Productivity in Austria between 1964 and 1980", Empirical Economics, Nr. 1, S. 27-49

Morehouse, W. (1985): "Beyond SuperStock. The Three-Tiered Plan for Universal Capital Ownership", Journal of Post Keynesian Economics, Nr. 3, S. 435-439

Mumford, L. (1934): Technics and Civilization, Hartcourt, New York

Neubauer, H. (1984): „Schwarzarbeit und Eigenleistungen in der österreichischen Bauwirtschaft“, in: Skolka, J. (Hrsg.): „Die andere Wirtschaft: Schwarzarbeit und Do-ityourself in Österreich", Signum, Wien, S. 131-160

Oates, M. I., Baumol, W. J. (1972): "On the Economics of Theater in Renaissance London", The Swedish Journal of Economics, Nr. 1, S. 136-160

OECD - Organization for Economic Cooperation and Development (1974): "Inflation and the Service Sector", OECD Observer, Nr. 12, S. 27-29

ÖIV - Österreichisches Institut für Verpackungswesen an der Wirtschaf tsuniversität Wien (1978): „10. Bericht-Selbstbedienung Österreich“, Wien

ÖIV - Österreichisches Institut für Verpackungswesen an der Wirtschaf tsuniversität Wien (1984): „16. Bericht-Selbstbedienung Österreich“, Wien

Opielka, M., Votruba, G. (1986): Das garantierte Grundeinkommen: Entwicklung und Perspektiven einer Forderung, Fischer, Frankfurt am Main

ÖStZ - Österreichisches Statistisches Zentralamt (1965): „Statistisches Handbuch für die Republik Österreich 1965“, ÖStZ, Wien

ÖStZ - Österreichisches Statistisches Zentralamt (1977): „Statistisches Handbuch für die Republik Österreich 1977“, ÖStZ, Wien

ÖStZ - Österreichisches Statistisches Zentralamt (1985): „Statistisches Handbuch für die Republik Österreich 1985“, ÖStZ, Wien

Pahl, R. E. (1984): "Divisions of Labour”, Blackwell, Oxford 
Paukert, F. (1973): "Income Distribution at Different Levels of Development: A Survey of Evidence”, International Labour Review, Nr. 2-3, S. 97-125

Pfannkuch, E. (1976): „Der Einfluß der Technisierung der Haushaltswirtschaft auf das Geld- und Zeitbudget ausgewählter Haushalte im wirtschaftlichen und sozialen Wandel“, in: Schweitzer, R. V., Pross, H. (Hrsg.): „Die Familienhaushalte im wirtschaftlichen und sozialen Wandel", Schwartz, Göttingen

Poapst, J. V., Waters, W. R. (1964): "Rates of Return on Consumer Durables", Journal of Finance, Dez., S. 673-677

Polanyi, K. (1944): “The Great Transformation", Beacon, Boston

Préel, B. (1986): Essai sur l'avenir des services: Quatre cas exemplaires, Final Report to the FAST Programme, EEC, Brüssel

Presvelou, C. (1986): Households, the Home Computer and Related Services in the Netherlands: Attitudes, Trends and Prospects, Final Report to the FAST Programme, EEC, Brüssel

Puwein, W. (1984): „Parallele Wirtschaft im Verkehr“, in: Skolka, J. (Hrsg.): Die andere Wirtschaft: Schwarzarbeit und Do-it-yourself in Österreich, Signum, Wien, 1984, S. $165-178$

Puwein, W. (1985): „Prognose des Personenverkehrs bis zum Jahr 2000“, Österreichisches Institut für Wirtschaftsforschung, Wien

Quesnay, F. (1758): Tableau oeconomique

Reid, M. (1934): Economics of Household Production, John Wiley and Sons, New York

Rinderspacher, J. P. (1985): Gesellschaft ohne Zeit, Campus, Frankfurt/Main

Robbins, L. (1932): The Nature and Significance of Economic Science, MacMillan, London

Schäfer, D. (1985): „Wert des Gebrauchsvermögens der privaten Haushalte“, Wirtschaft und Statistik, Nr. 2, S. 110-118

Schaff, A. (1985): Wohin führt der Weg? Die gesellschaftlichen Folgen der zweiten industriellen Revolution, Europa Verlag, Wien

Scharpf, F. W. (1985): „Strukturen der post-industriellen Gesellschaft" Wirtschaft und Gesellschaft, Nr. 1, S. 9-34

Schneider, M. (1984): „Eigenleistungen in der Land- und Forstwirtschaft“, in: Skolka, J. (Hrsg.): Die andere Wirtschaft - Schwarzarbeit und Do-it-yourself in Österreich, Signum, Wien, S. 219-229

Schumpeter, J. A. (1954): History of Economic Analysis, George Allen and Unwinn Ltd., London

Scitovski, T. (1972): "What's Wrong with the Arts is What's Wrong with Society", The American Economic Review, Nr. 2, S. 62-69

Simhandl, G. (1984): „Der Tagesablauf der Österreicher“, in: Skolka, J. (Hrsg.): Die andere Wirtschaft - Schwarzarbeit und Do-it-yourself in Österreich, Signum, Wien, S. 39-58

Skolka, J. (1976a): "Long-Term Effects of Unbalanced Labour Productivity Growth: On the Way to a Self-Service Society", in: Solari, L., Pasquier, J.-N. (Hrsg.): Private and Enlarged Consumption: Essays in Methodology and Empirical Analysis, North-Holland, Amsterdam, S. 279-301

Skolka, J. (1976b): "The Substitution of Self-Service Activities for Marketed Services", The Review of Income and Wealth, Nr. 4, S. 297-304

Skolka, J. (1977): „Dienstleistungen oder Selbstbedienung“, Wirtschaft und Gesellschaft, Nr. 4, S. 397-406

Skolka, J. (1985): „Wende in der Arbeitsteilung“, Wirtschaft und Gesellschaft, Nr. 4, S. $445-469$

Skolka, J. (1986): „Der Dienstleistungssektor der österreichischen Wirtschaft“, WIFOMonatsberichte, Nr. 10, S. 584-611

Skolka, J. (1987): „Wissen, Arbeitsteilungen und Strukturwandel“, Wirtschaft und Gesellschaft, Nr. 2, S. 245-270

Smith, A. (1776): An Inquiry into the Nature and the Causes of the Wealth of Nations, Edition Clarendon Press, Oxford, 1979

Speiser, S. M. (1985): "Broadened Capital Ownership - The Solution to Major Domestic and International Problems", Journal of Post Keynesian Economics, Nr. 3, S. 426-434

Summers, R., (1985): "Services in the International Economy", in: Inman, R. P. (Hrsg.): Managing the Service Economy: Prospects and Problems, Cambridge University Press, Cambridge, S. 27-48 
Theobald, R. (1965): Free Men and Free Markets, Doublheday-Anchor, New York

Thienen, V. von (1986): Zeitstruktur und technisch-gesellschaftlicher Wandel, Wissenschaftszentrum Berlin, Berlin

Thompson, E. P. (1971): "The Moral Economy and the English Crowd in the 18th Century", Past and Present, Nr. 50, S. 76-136

Tullock, G. (1974): "On the Economics of Theater in Renaissance London and Gay Nineties Eldora", The Swedish Journal of Economics, Nr. 3, S. 366-368

Usher, P. J. (1980): A northern Perspective on the Informal Economy, The Vanier Institute of Family, Ottawa

Vocelka-Zeidler, S. (1985): „Ausstattung der Haushalte“, Statistische Nachrichten, Nr. 7, S. $476-483$

Weinrobe, M. (1974): "Household Production and National Production: An Improvement of the Record", The Review of Income and Wealth, Nr. 1, S. 89-102

Wüger, M. (1981): „Dauerhafte Konsumgüter - Nachfrage und Ausstattung der österreichischen Haushalte“, in: WIFO Monatsberichte, Nr. 2, S. 63-78 